\title{
Density of Langerhans cells in chronic plaque psoriatic lesions before and after phototherapy
}

\author{
HELENA ROTSZTEJN ${ }^{\prime}$, DOROTA CHOMICZEWSKA², EWA TRZNADEL-GRODZKA ${ }^{3}$, \\ DOROTA JESIONEK-KUPNICKA ${ }^{4}$ \\ ${ }^{1}$ Department of Cosmetology, Medical University of Lodz, Poland \\ ${ }^{2}$ Nofer Institute of Occupational Medicine, Centre of Occupational Allergy and Environmental Health, Lodz, Poland \\ ${ }^{3}$ Department of Dermatology, Paediatric Dermatology and Dermatological Oncology, Medical University of Lodz, Poland \\ ${ }^{4}$ Department of Tumor Pathology, Medical University of Lodz, Poland
}

\begin{abstract}
Introduction: Langerhans cells (LCs) constitute a key component of the skin immune system (SIS). They play some role in the pathogenesis of chronic inflammatory diseases of the skin, including psoriasis. However, their role in psoriasis has not been completely elucidated. The aim of this study was to evaluate LCs density in skin lesions of patients with chronic plaque psoriasis, before and after phototherapy.

Material and methods: 25 patients with plaque psoriasis were included in the study. Biopsies were taken from the skin of their lumbosacral or gluteal area. Then the participants received broadband UVB, narrowband UVB or PUVA therapy. Immunohistochemical staining was performed on formalin-fixed paraffin-embedded skin samples using antihuman CDla antibody (NCL-CD1a-235, Novocastra).

Results: The number of LCs in the study group was lower in lesional skin compared to perilesional skin. After phototherapy we observed an increase in the number of LCs in lesional skin while a decrease of the LCs number was noted in perilesional skin after PUVA treatment.

Conclusions: Psoriatic epidermis is characterized by disturbances in the number of LCs. Our study showed a low number of LCs in chronic psoriatic plaques. At the same time, a high number of LCs in perilesional skin may predict centrifugal enlargement of psoriatic plaques. However, a smaller number of LCs may be observed in chronic lesions. The most considerable increase of LCs number was observed in psoriatic skin of our patients after BB-UVB and PUVA treatment, a slight increase - after NB-UVB therapy.
\end{abstract}

Key words: Langerhans cells, psoriasis, phototherapy, CDIa.

(Centr Eur J Immunol 2012; 37 (3): 258-263)

\section{Introduction}

Langerhans cells (LCs) are members of the dendritic cell family, residing in the basal and suprabasal layers of the epidermis. They belong to the skin immune system (SIS) and specialize in antigen presentation. LCs acquire antigens in the skin, transport them to regional lymph nodes, present to naive $\mathrm{T}$ cells and initiate adaptive immune response [1]. LCs have strong immunogenic properties but they may also act as mediators of tolerance, for example to commensal bacteria $[2,3]$. They are involved in antimicrobial immunity, skin immunosurveillance and induction phase of the contact hypersensitivity $[1,2]$. They are also likely to play some role in the pathogenesis of chronic inflammatory diseases of the skin, including psoriasis.

Psoriasis is a chronic skin disease that affects about $1.5 \%$ of the Caucasian population and manifests itself by typical macroscopic and microscopic skin alterations [4]. It is now recognized as a common immune-mediated disorder [5]. Since the 1990s, type 1 T cells have been thought to play a dominant role in initiation and maintenance of psoriasis. Recently it has been postulated that the pathogenesis of psoriasis consists of distinct subsequent stages, in each of them different cell types play an important role. At the onset of psoriasis, the initial step may comprise the 
recognition and uptake of antigens by tissue-guarding, immature dendritic cells (DCs) and presentation of antigens to T cells (streptococcal, other exogenous and endogenous antigens) [4]. The contribution of LCs to the evolution of psoriasis has not been completely elucidated among other subsets of DCs [6, 7]. Reports concerning the LCs number in lesional and non-lesional skin of individuals with psoriasis were variable [8-10].

There is an abundant evidence that UVR induces LCs apoptosis, necrosis or migration from the epidermis to the regional lymph nodes, all of them resulting in decrease of LCs density in epidermis [11-13]. The effect of different methods of phototherapy on LCs in psoriasis was investigated in a certain number of studies, showing significant or non-significant decrease in the LCs frequency in the skin of psoriatic patients after treatment [14-18].

The aim of our study was to evaluate the number of LCs in chronic plaque psoriatic skin and to assess the impact of phototherapy on the number of LCs in the same location. In some cases we evaluated LCs in perilesional skin as well.

\section{Material and methods}

\section{The study group}

25 individuals suffering from plaque psoriasis were included in the study, after giving written informed consent. They were randomly selected from patients referred to Phototherapy Unit of Dermatology Department of Medical University in Lodz, Poland. The study was performed after obtaining approval of the suitable ethical committee. All patients, 13 males and 12 females, aged from 21 to 75, medium age 46, were white Caucasians of phototype II or III. They had not undergone systemic immunosupressive treatment nor phototherapy during six months prior to the study. The PASI scores ranged from 4.8 to 17.8 , medium 10.13 .

Four or three mm punch biopsies were taken from the skin of the lumbosacral or gluteal area: from psoriatic lesions in some participants and from perilesional uninvolved skin in some of them, approximately $1 \mathrm{~cm}$ from the border of the plaque. Then one of three methods of phototherapy was performed in participants: broadband UVB (BB-UVB, initial dose $0.02 \mathrm{~J} / \mathrm{cm}^{2}$ ), narrowband UVB (NB-UVB initial dose $0.1 \mathrm{~J} / \mathrm{cm}^{2}$ ) or PUVA (with oral intake of methoxsalen, $20 \mathrm{mg}$ per $\mathrm{kg}, 2$ hours before UVA irradiation, initial dose of UVA

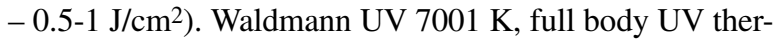
apy system was used as a source of BB-UVB and UVA, the spectrum of UVB wave-length was 280-360 nm and UVA - 320-400 nm; NB-UVB therapy was performed using PUVA Combi Light Cabin/PCL 8000 Full Body System, with TL01 lamp emitting radiation at $312 \mathrm{~nm}$.

The aim of the phototherapy was to obtain PASI 75 but some individuals decided to finish the treatment earlier. All subjects were asked not to apply topical treatment to the skin area adjoining the site of biopsies. 18 patients agreed on taking skin biopsies from lesional or perilesional skin of the same area after termination of the phototherapy and within a few hours after the last irradiation. 8 of them underwent UVB-BB treatment, 7 - NB-UVB and 3 - PUVA treatment.

\section{Immunohistochemistry}

Immunohistochemical staining was performed on formalin-fixed paraffin-embedded skin samples using antihuman CD1a antibody (NCL-CD1a-235, Novocastra). This antibody recognizes the human CD1a cell surface glycoprotein, a 43-49 $\mathrm{kDa}$ molecule expressed in association with $\beta_{2}$-microglobulin. CD1a is expressed strongly by cortical thymocytes, LCs in the epidermis and dendritic cells in dermis.

The paraffin-embedded sections of tumor were deparaffinized, rehydrated, and heat-treated for citrate microwave antigen retrieval in $10 \mathrm{mM}$ citrate buffer, $\mathrm{pH} \mathrm{6.0,} \mathrm{at}$ $350 \mathrm{~W}$ for $15 \mathrm{~min}$ and then cooled to room temperature. Sections were then blocked for peroxidases in $0.3 \% \mathrm{H}_{2} \mathrm{O}_{2}$ in methanol for $30 \mathrm{~min}$, and incubated with the primary antibody anti-CD1a (dilution $1: 50$ ) for $30 \mathrm{~min}$ at room temperature in a humidity chamber. For detection, a Dako Envision System HRP with DAB staining and hematoxylin counterstaining was used. For a negative control, the slides were stained with the omission of the primary antibody.

\section{Evaluation of LCs}

Immunohistochemical staining revealed that LCs exhibited characteristic processes. The number of LCs in each of the cases was estimated as the number of cells calculated per 10 high-power fields (HPFs) at 200× magnification.

\section{Results}

The number of LCs in psoriatic lesional skin was evaluated in 25 individuals and in perilesional area in 20 of them (several patients did not agree to another excision). Results of evaluation are showed in Table 1 . The number of LCs in lesional skin was lower compared to perilesional skin

Table 1. The number of Langerhans cells in lesional skin of 25 individuals with plaque psoriasis and perilesional skin of 20 individuals with plaque psoriasis (number/10 HPF)

\begin{tabular}{lccccc}
\hline Skin area & $\boldsymbol{n}$ & Range & Mean & Median & SD \\
\hline lesional & 25 & $0.0-32.0$ & 6.53 & 7.56 \\
\hline perilesional & 20 & $3.0-46.0$ & 19.32 & 17.5 \\
\hline
\end{tabular}


A

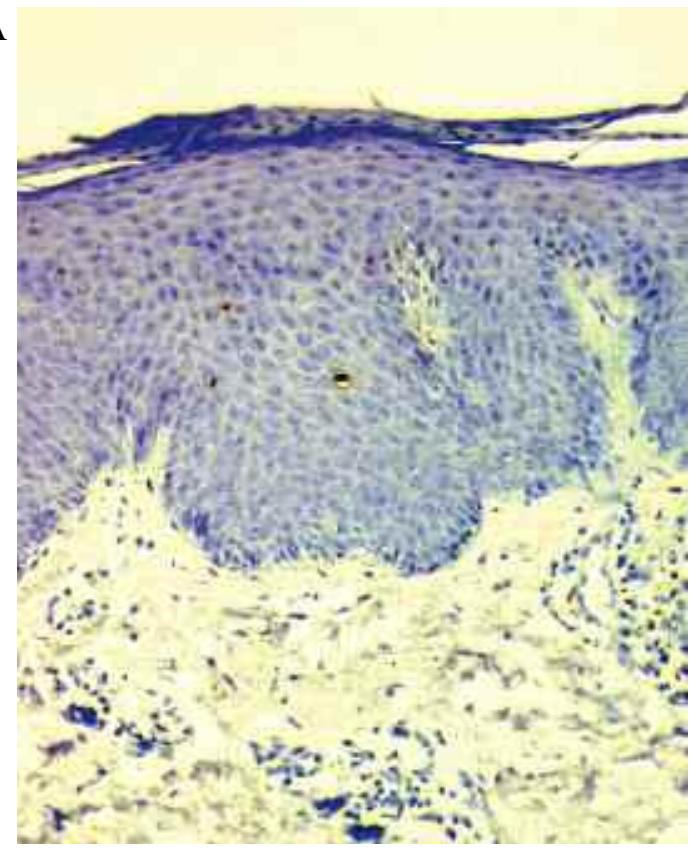

B

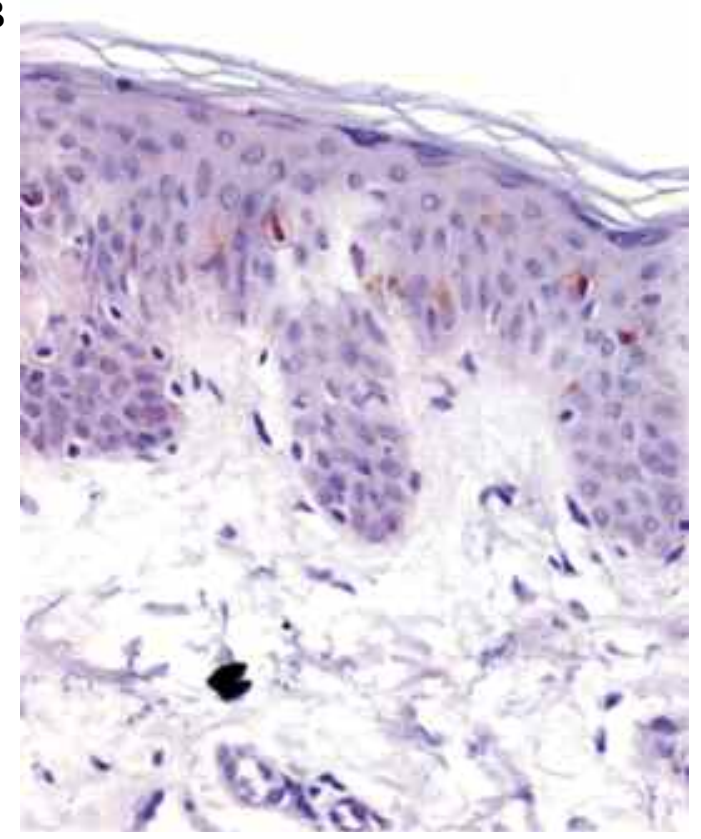

Fig. 1. Before phototherapy: A - the low number of CD1a positive Langerhans cells (LCs) in plaque psoriasis; B - the highest number of CD1a positive LCs in perilesional skin compared with lesional skin, anti-CD1a immunohistochemistry, hematoxylin counterstaining $200 \times$

Table 2. The mean number of Langerhans cells in lesional and perilesional skin of individuals with plaque psoriasis before and after UVB BB and PUVA therapy (number/10 HPF)

\begin{tabular}{lcccc}
\hline Phototherapy & $\boldsymbol{n}$ & Skin area & Before \pm SD $($ range $)$ & After \pm SD (range) \\
\hline UVB BB & 8 & lesional & $2.33 \pm 2.57(0.0-7.0)$ & $10.78 \pm 8.48(3.0-29.0)$ \\
\cline { 2 - 4 } & & perilesional & $13.77 \pm 5.61(8.0-22.0)$ & $16.57 \pm 9.48(4.0-31.0)$ \\
\hline PUVA & 3 & lesional & $3.33 \pm 2.52(1.0-6.0)$ & $14.67 \pm 7.02(8.0-22.0)$ \\
\cline { 2 - 4 } & & perilesional & $26.67 \pm 10.41(15.0-35.0)$ & $17.67 \pm 3.21(14.0-20.0)$ \\
\hline UVB 311 & 4 & lesional & $10.3 \pm 3.38(7.0-13.0)$ & $13.65 \pm 4.38(8.0-18.0)$ \\
\hline
\end{tabular}

(Fig. 1). The comparative assessment of LCs number in chronic plaque psoriatic skin before and after phototherapy was conducted in 8 patients who underwent BB-UVB (dose range: 0.14-4.16 J/cm², medium $1.87 \mathrm{~J} / \mathrm{cm}^{2}$ ), 3 patients who underwent PUVA treatment (dose range: $10-25.25 \mathrm{~J} / \mathrm{cm}^{2}$, medium $18.25 \mathrm{~J} / \mathrm{cm}^{2}$ and 4 patients after NB-UVB therapy (dose range: $3.25-8.6 \mathrm{~J} / \mathrm{cm}^{2}$, medium $5.42 \mathrm{~J} / \mathrm{cm}^{2}$ ) (3 patients were excluded from the study despite taking biopsies: two because of technical reasons and one because of extremely high number of LCs).

The number of LCs in previously lesional skin was higher after all BB-UVB, NB-UVB and PUVA irradiation compared to the number before phototherapy. Results of the comparison are showed in Table 2.

The comparative assessment of LCs number in perilesional skin before and after phototherapy was performed in
8 individuals who underwent BB-UVB (dose range: $0.4-4.16 \mathrm{~J} / \mathrm{cm}^{2}$, medium $2.11 \mathrm{~J} / \mathrm{cm}^{2}$ ) and in 3 patients who underwent PUVA treatment (dose range: $10-25.25 \mathrm{~J} / \mathrm{cm}^{2}$, medium $18,25 \mathrm{~J} / \mathrm{cm}^{2}$ ). The count of LCs in perilesional skin was comparable before and after BB-UVB treatment and lower after PUVA therapy compared to the number before the treatment (Table 2, Fig. 2).

\section{Discussion}

Our results showed a low number of epidermal LCs in psoriatic plaques in comparison to surrounding non-lesional skin. In a few studies similar results were observed $[19,20]$. Komine et al. found a significant increase in LCs density in perilesional skin of psoriatic plaques which may suggests their further roles in the formation of plaques [21]. 
A

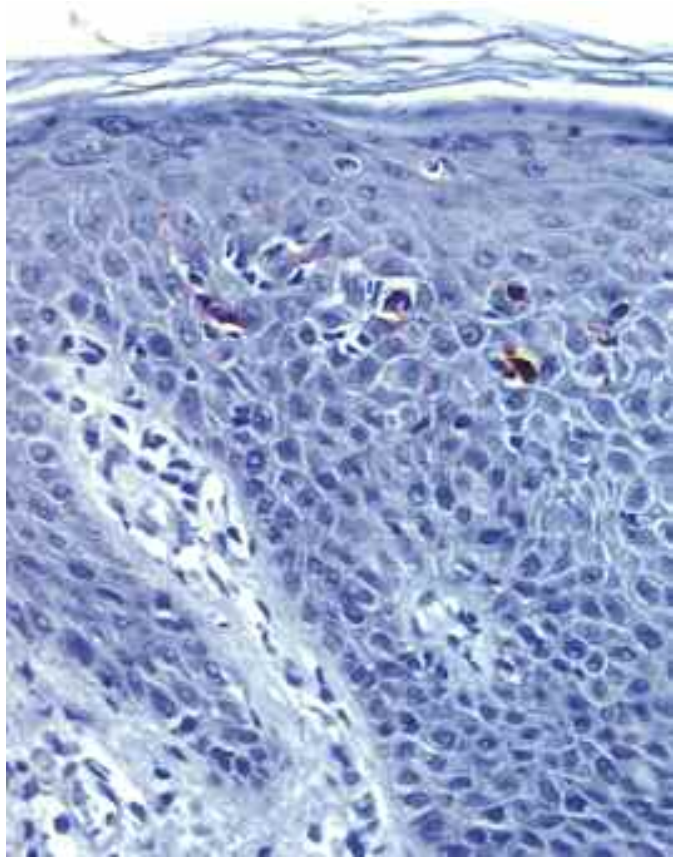

B

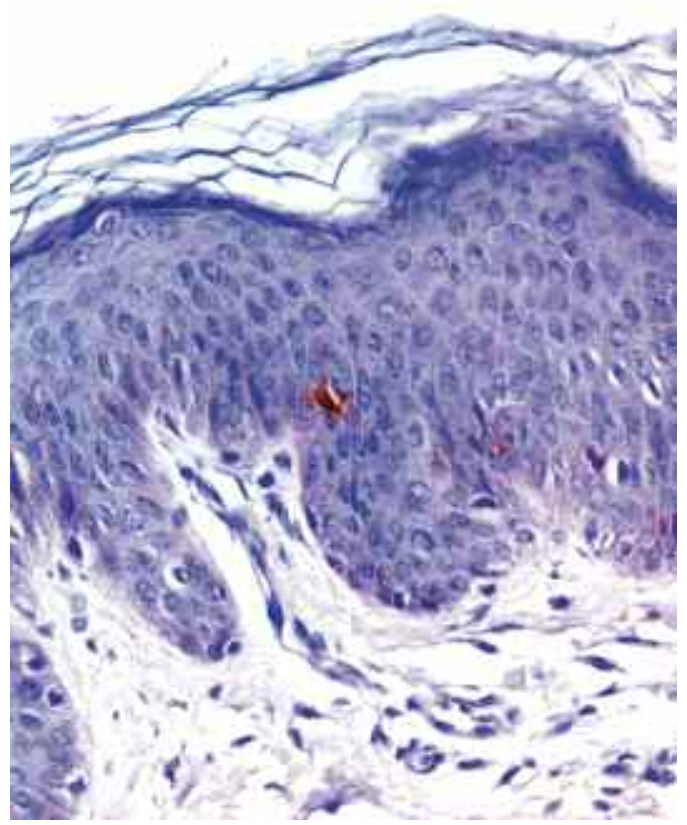

Fig. 2. A - the number of CD1a positive LCs in previously lesional skin after phototherapy was higher compared to the number of LCs before phototherapy; B - CD1a positive LCs in perilesional skin after phototherapy, anti-CD1a immunohistochemistry, hematoxylin counterstaining $200 \times$

Deguchi et al. revealed the lack of difference in the number of LCs between psoriatic and normal skin [22]. In a few studies the count of LCs in psoriatic lesions was increased in comparison to uninvolved or healthy skin $[8,21,23]$.

Immunohistochemical analysis of different stages of cellular infiltrate development in psoriasis showed a higher number of CD1a+ cells in chronic changes [24], but there are also studies which support the hypothesis of increased LCs density in early changes [21].

The reasons of these variable reports have been discussed by some authors. The number of LCs in lesional skin may differ depending on the phase of development of plaque, its time of duration, localization [21] and also a type of psoriasis (guttate or plaque) [8]. Different staining and counting methods, nonequivalent patients populations and differences in structure of healthy epidermis and acanthotic psoriatic epidermis may also influence the results [20, 25, 26].

We assessed skin biopsies taken from patients with chronic plaque psoriasis. Our results showed a decreased number of LCs in psoriatic skin whereas in the surrounding tissue the number of LCs was high. An increased number of LCs in perilesional skin may reflect asymptomatic changes in the SIS preceding potential centrifugal enlargement of the lesions [21]. It is thought that local skin-resident immune cells (including DCs) and associated cytokines are present in the uninvolved skin of psoriatic patients and may be sufficient to initiate or maintain lesions following inciting triggers [27]. At the same time chronic lesions may contain a smaller number of LCs. Yamanaka et al. showed that there was a decreased number of LCs in the atrophic centre of the lesions of anular lichen planus [28]. Rotsztejn et al. showed a small number of LCs of vulvar lichen sclerosus in the late atrophic stage [29].

In several studies the number of LC in lesional or noninvolved skin of psoriatic patients was reduced or almost depleted after systemic PUVA treatment in several studies [15, 30-36]. Similar effects were noted after PUVA-bath therapy (both using trimethylpsoralen and methoxypsoralen) $[14,35,37]$ which resulted in a decrease in LCs number in psoriatic epidermis. It is assumed that the reduction in epidermal LCs may be one of the factors contributing to therapeutic action of systemic PUVA and PUVA-bath treatment in patients with psoriasis.

In a few studies, effect of UVB irradiation on epidermal CD1a+ cells in psoriatic skin was investigated. DeSilva et al. [18] reported that TL01 therapy (NB-UVB) induced twofold decrease in LCs number whereas according to Erkin et al. [15] NB-UVB irradiation did not change epidermal LCs count in psoriatic skin. In healthy volunteers, a significant decrease in epidermal LCs count was observed post broad- and narrowband UVB exposure, without a significant difference in effect between them [38]. In previous studies, BB-UVB resulted in stronger reduction in LCs density in murine epidermis than NB-UVB irradiation [39]. 
According to numerous data pointing at depletion of epidermal LCs following UV exposure, we expected a similar effect in our patients after UVB and PUVA therapy. However, we observed an increase of LCs count in lesional skin after phototherapy. In our opinion, appearance of LCs was related to a reversal of typical features of psoriatic epidermis as hyperkeratosis, parakeratosis and acanthosis accompanied by disturbed distribution of cells of the SIS. The return to the normal organization of epidermis resulted in reappearance of LCs - common constituent of basal and suprabasal layers. Similar effect was observed in psoriatic lesional skin after adalimumab - TNF- $\alpha$ inhibitor [40] and cyclosporine A [41], however Prignano et al. [42] reported a $70 \%$ decrease of LCs number in psoriatic plaques 4 months after biological therapies. Normalization of the distribution of LCs in psoriasis was reported after systemic aromatic retinoid i.e. etretinate administration [19]. Moderate decrease in LCs count in perilesional skin after PUVA treatment is consent with results of most previous studies and may also reflect normalization of immunological state of the skin.

\section{Conclusions}

In our study psoriatic epidermis was characterized by disturbances in the number of LCs with their strikingly low number in chronic psoriatic lesions and a comparably high number in perilesional skin. A high number of LCs in perilesional skin may predict centrifugal enlargement of psoriatic plaques. However, chronic lesions may contain a smaller number of LCs. The most considerable increase of LCs number was observed in psoriatic skin after BBUVB and PUVA treatment, a slight increase - after NBUVB therapy.

\section{References}

1. Valladeau J, Saeland S (2005): Cutaneous dendritic cells. Semin Immunol; 17: 273-283

2. Berger CL, Vasquez JG, Shofner J, et al. (2006): Langerhans cells: mediators of immunity and tolerance. Int J Biochem Cell Biol 38: 1632-1636.

3. Van Der Aar AM, Sylva-Steenland RM, Bos JD, et al. (2007): Cutting edge: loss of TLR2, TLR4, and TLR5 on Langerhans cells abolishes bacterial recognition. J Immunol 178: 1986-1990.

4. Sabat R, Philipp S, Höflich C, et al. (2007): Immunopathogenesis of psoriasis. Exp Dermatol 16: 779-798.

5. Griffiths CE, Barker JN (2007): Pathogenesis and clinical features of psoriasis. Lancet 370: 263-271.

6. Jariwala PS (2007): The role of dendritic cells in the immunopathogenesis of psoriasis. Arch Dermatol Res 299: 359-366.

7. Johnson-Huang LM, Scott McNutt N, et al. (2009): Cytokineproducing dendritic cells in the pathogenesis of inflammatory skin diseases. J Clin Immunol 29: 247-256.

8. Ashworth J, Mackie RM (1986): A quantitative analysis of the Langerhans cell in chronic plaque psoriasis. Clin Exp Dermatol 11: 594-599.
9. Ramirez-Bosca A, Martinez-Ojeda L, Valcuende-Cavero F, Castells-Rodellas A (1988): A study of local immunity in psoriasis. Br J Dermatol 119: 587-595.

10. Bieber T, Braun-Falco O. Distribution of CD1a-positive cells in psoriatic skin during the evolution of the lesions. Acta Derm Venereol 1989; 69: 175-178.

11. Nakagawa S, Koomen CW, Bos JD, Teunissen MB. Differential modulation of human epidermal Langerhans cell maturation by ultraviolet B radiation. J Immunol 1999; 163: 5192-5200.

12. Seité S, Zucchi H, Moyal D, et al. Alterations in human epidermal Langerhans cells by ultraviolet radiation: quantitative and morphological study. Br J Dermatol 2003; 148: 291-299.

13. Norval M. The mechanisms and consequences of ultravioletinduced immunosuppression. Prog Biophys Mol Biol 2006; 92: 108-118.

14. Coven TR, Murphy FP, Gilleaudeau P, et al. (1998): Trimethylpsoralen bath PUVA is a remittive treatment for psoriasis vulgaris. Arch Dermatol 1998; 134: 1263-1268.

15. Erkin G, Uğur Y, Gürer CK, et al. (2007): Effects of PUVA, narrow band UVB and cyclosporine on inflammatory cells of the psoriatic plaque. J Cutan Pathol 2007; 34: 213-219.

16. Malinowska K, Sysa-Jędrzejowska A, Woźniacka A (2011): UVA1 phototherapy in dermatological treatment. Post Dermatol Alergol 28: 53-58.

17. Wojciech Silny W, Agnieszka Osmola-Mańkowska A, Magdalena Czarnecka-Operacz M, Aleksandra Szewczyk A (2010): Wąskozakresowa fototerapia UVA-1 w lecznictwie dermatologicznym - pierwsze polskie doświadczenia. Post Dermatol Alergol 2010; 27: 1-10.

18. DeSilva B, McKenzie RC, Hunter JAA, Norval M (2008): Local effects of TL01 phototherapy in psoriasis. Photodermatol Photoimmunol Photomed 24: 268-269.

19. Haftek M, Faure M, Schmitt D, Thivollet J (1983): Langerhans cells in skin from patients with psoriasis: Quantitative and qualitative study of T6 and HLA-Dr antigen-expressing cells and changes with aromatic retinoid administration. J Invest Dermatol 81: 10-14.

20. Bieber T, Ring J, Braun-Falco O (1988): Comparison of different methods for enumeration of Langerhans cells in vertical cryosections of human skin. Br J Dermatol 118: 385-392.

21. Komine M, Karakawa M, Takekoshi T, et al. (2007): Early inflammatory changes in the "perilesional skin" of psoriatic plaques: is there interaction between dendritic cells and keratinocytes? J Invest Dermatol 127: 1915-1922.

22. Deguchi M, Aiba S, Ohtani H, et al. (2002): Comparison of the distribution and numbers of antigen-presenting cells among T-lymphocyte-mediated dermatoses: CD1a+, factor XIIIa+, and CD68+ cells in eczematous dermatitis, psoriasis, lichen planus and graft-versus-host disease. Arch Dermatol Res 294: 297-302.

23. Baker BS, Swain AF, Griffiths CE, et al. (1985): Epidermal T lymphocytes and dendritic cells in chronic plaque psoriasis: the effects of PUVA treatment. Clin Exp Immunol 61: 526-534.

24. Onuma S (1994): Immunohistochemical studies of infiltrating cells in early and chronic lesions of psoriasis. J Dermatol 1994; 21: 223-232.

25. Jones IL, Berth-Jones J, Fletcher A, Hutchinson PE (1994): Assessment of epidermal dendritic cell markers and T-lymphocytes in psoriasis. J Pathol 174: 77-82.

26. Zaba LC, Krueger JG, Lowes MA (2009): Resident and "inflammatory" dendrtic cells in human skin. J Invest Dermatol 129: 302-308.

27. Boyman O, Conrad C, Tonel G, et al. (2007): The pathogenetic role of tissue-resident immune cells in psoriasis. Trends Immunol 28: 51-57. 
28. Yamanaka Y, Akiyama M, Shibaki A, et al. (2004): Annular lichen planus: study of the cellular mechanisms of annularity. Dermatology 208: 335-338.

29. Rotsztejn H, Trznadel-Budźko E, Jesionek-Kupnicka D (2006): Langerhans cells in vulvar lichen sclerosus and vulvar squamous cell carcinoma. Arch Immunol Ther Exp (Warsz) 54: 363-366.

30. Friedmann PS (1981): Disapperance of epidermal Langerhans cells during PUVA therapy. Br J Dermatol 105: 219-221.

31. Ree K (1982): Reduction of Langerhans cells in human epidermis during PUVA therapy: a morphometric study. J Invest Dermatol 78: 488-492.

32. Friedmann PS, Ford G, Ross J, Diffey BL (1983): Reappearance of epidermal Langerhans cells after PUVA therapy. $\mathrm{Br}$ J Dermatol 109: 301-307.

33. Koulu L, Söderström KO, Jansén CT (1984): Relation of antipsoriatic and Langerhans cell depleting effects of systemic psoralen photochemotherapy: a clinical, enzyme histochemical, and electron microscopic study. J Invest Dermatol 82: 591-593.

34. Bos J, Krieg SR (1985): Psoriasis infiltrating cell immunophenotype: changes induced by PUVA or corticosteroid treatment in T-cell subsets, Langerhans' cells and interdigitating cells. Acta Derm Venereol 65: 390-397.

35. Czernielewski J, Juhlin L, Shroot S, Brun P (1985): Langerhans' cells in patients with psoriasis: effect of treatment with PUVA, PUVA bath, etretinate and anthralin. Acta Derm Venereol 1985; 65: 97-101.

36. Torinuki W, Mauduit G, Haftek M, Thivolet J (1987): Effects of PUVA and mechlorethamine treatment of psoriatic patients on epidermal Langerhans' cells. Acta Derm Venereol 67: 532-535.

37. Piérard-Franchimont C, Nickels-Read D, Ben Mosbah T, et al. (1990): Early dermato-pathological signs during bath-PUVA therapy. J Pathol 161: 227-231.

38. Tijoe M, Smits T, van der Kerkhof PCM, Gerritsen MJ (2003): The differential effect of broad band vs. narrow band UVB with respect to photodamage and cutaneous inflammation. Exp Dermatol 12: 729-733.

39. El-Ghorr AA, Norval M (1997): Biological effects of narrowband (311 nm TL01) UVB irradiation: a review. J Photochem Photobiol B 38: 99-106.

40. Gordon KB, Bonish BK, Patel T, et al. (2005): The tumor necrosis factor- $\alpha$ inhibitor adalimumab rapidly reverses the decrease in epidermal Langerhans cell density in psoriatic plaques. Br J Dermatol 153: 945-953.

41. Horrocks C, Duncan JI, Sewell HF, et al. (1990): Differential effects of cyclosporine A on Langerhans cells and regulatory T-cell populations in severe psoriasis: an immunohistochemical and flow cytometric analysis. J Autoimmun 3: 559-570.

42. Prignano F, Ricceri F, Bianchi B, Lotti T (2009): Quantity, distribution and immunophenotypical modification of dendritic cells upon biological treatments in psoriasis. Int J Immunopathol Pharmacol 22: 379-387. 